\title{
EVALUACIÓN DE LA FATIGA LABORAL EN CONDUCTORES DE LA COOPERATIVA DE TRANSPORTE DEL MUNICIPIO DE PLANADAS
}

\author{
Jacid Montoya-Torres $^{1 *}$, Daydu Milena Robayo-Barrios ${ }^{2}$ y Sandra Patricia Monroy-Caicedo ${ }^{3}$ \\ 1,2,3 Corporación Universitaria Minuto de Dios, Ibagué, Colombia \\ *Autor de correspondencia: jacid.montoya@uniminuto.edu.co
}

Recibido Octubre 2020; Aceptado Diciembre 2020

\begin{abstract}
Resumen - Las posturas prolongadas son la principal causa de carga estática en el sistema musculoesquelético humano y, generalmente, están asociadas al trabajo. Según estudios epidemiológicos, mantener una postura por un tiempo determinado genera fatiga, molestias y dolor muscular. En Colombia las investigaciones relacionadas a la carga postural en conductores son limitadas, a pesar de que las Administradoras de Riesgos Laborales y la Federación Colombiana de Transportadores de Carga por Carretera demostraron que existe incompatibilidad entre las características físicas de los conductores y los puestos en que desempeñan su trabajo (Pachón, Barrios y Pérez, 2017, p.51); lo que podría ocasionar malestares físicos y alteraciones en el estado de ánimo, entre otros síntomas. En el departamento del Tolima, por ejemplo, los conductores de servicio público desarrollan extensas jornadas de trabajo que exigen máxima concentración y esfuerzo y que se prolongan desde la madrugada hasta cubrir la totalidad de las rutas asignadas, a altas horas de la noche. En este sentido, la presente investigación aborda las condiciones en que se genera fatiga laboral en conductores de la zona de consolidación del sur del departamento del Tolima; con el propósito de documentar los agentes etiológicos y los factores de riesgo que se presentan en la Cooperativa de Transportadores del municipio de Planadas. El proyecto se orienta a diseñar mecanismos de prevención de accidentes y enfermedades laborales en el objeto práctico de estudio.
\end{abstract}

Palabras clave: Fatiga laboral, condiciones de trabajo, conductores, transporte público, posturas prolongadas.

\section{LABORAL FATIGUE EVALUATION IN DRIVERS OF THE COOPERATIVA DE TRANSPORTE OF PLANADAS MUNICIPALITY}

\begin{abstract}
Prolonged postures are the most common cause of static load in the human muscle-skeletal system and, generally, they are related to work. Epidemiological studies say that staying in a position for a determined time generates fatigue, discomfort, and muscular pain. In Colombia, research on postural load in drivers is limited, although Laboral Risk Managers and Federación Colombiana de Transportadores de Carga por Carretera have demonstrated that exist an incompatibility between physical characteristics of drivers and their working spots (Pachón, Barrios y Pérez, 2017, p.51). This situation could generate discomfort, pain, and alterations of their state of mind. In Tolima, for instance, public service drivers work during extended journeys with high effort and concentration, from dawn until late night. In this sense, the present study deals with conditions of occupational
\end{abstract}


fatigue in drivers from south of Tolima; with the purpose of registering etiological agents and risk factors of Cooperativa de Transportadores del municipio de Planadas. This project pretends to design prevention mechanisms for possible accidents and diseases in the study object.

Keywords: Laboral fatigue, work conditions, drivers, public transport, prolonged postures.

\section{Introducción}

La demanda de actividad productiva es un mecanismo que tiene el ser humano para garantizar su progreso físico, económico, social, espiritual y psicológico. Sin embargo, existe la posibilidad de que este mecanismo suscite una variación en la salud del individuo que comprometa su integralidad personal, administrativa y laboral (Vega-Gómez, Ceballos-Burgos y PardoCarmona, 2020); y que se manifieste a través de fenómenos tales como el agotamiento físico o emocional.

Precisamente, Lerman et al. (2012) sostiene que la fatiga es una respuesta natural del cuerpo humano a la falta de descanso o al esfuerzo prolongado que puede no ser solo físico: el concepto de fatiga laboral aborda el agotamiento mental y emocional al que se exponen las personas en su entorno de trabajo. Según The National Institute for Occupational Safety and Health (NIOSH) Science Blog (2020), la única manera de mitigar este este problema es proporcionando suficiente descanso al cuerpo.

Así pues, la higiene del sueño, la carga laboral, las condiciones de trabajo y las relaciones interpersonales han alcanzado una proporción de la población tan amplia, que se le considera un problema de salud pública. Incluso, la citada fuente destaca que en 2017, la conducción de vehículos por parte de personas en estado de somnolencia fue un factor determinante en la generación de 91.000 accidentes de tránsito, solo en los Estados Unidos (National Highway Traffic Safety Administration, 2016).

Por su parte, Colombia reportó 518 muertes por accidentes de tránsito en el año 2018, únicamente en su ciudad capital (Cámara de Comercio de Bogotá, 2019) y, si bien son muchos factores los que influyen en la seguridad vial de una región, cobra especial interés la incompatibilidad entre las condiciones y características físicas propias de los conductores y los puestos en los que desempeñan su trabajo (Pachón, Barrios y Pérez, 2017, p.51).

En el municipio de Planadas, al sur del Tolima, la Cooperativa de Transportadores cuenta con 144 conductores quienes se dedican al transporte especial colectivo de pasajeros urbano e intermunicipal. Su jornada laboral se desarrolla en el horario de ocho (8) horas diarias y que, según los requerimientos del servicio, se pueden extender hasta más de 12 horas. En esta medida, observar, evaluar y analizar las manifestaciones de fatiga patológica en esta población permitirá determinar los factores de riesgo asociados a su labor y generar estrategias de prevención orientadas a mitigar peligros e impactar positivamente su calidad de vida y rendimiento laboral. 


\section{Marco de referencia}

Existen factores que intervienen en las relaciones establecidas entre el ser humano y los sistemas o artefactos disponibles para la ejecución de procesos o interacciones de carácter laboral. Al estudio de estos factores y de la manera en que el entorno físico, ambiental y social afecta a las dos partes de esta relación, se le conoce como ergonomía (Madrid y Cañas, 2015, p.3). De acuerdo con la postura de Cruz \& Garnica (2010), el ambiente que rodea al operario afecta su desempeño en la utilización de los medios de trabajo o artefactos a su disposición. Por consiguiente, el comportamiento del individuo se ve influenciado por factores sociológicos tales como los mandos, el espacio vital, territorialidad, status, aprobación social, seguridad o conducta de grupo.

En este contexto, la psicología ergonómica se encarga de estudiar las reacciones y comportamientos individuales implicados en la naturaleza de la mente humana, que puedan tener incidencia en la interacción entre el individuo y su trabajo. Objeto de esta disciplina es el comportamiento social del individuo definido por factores psicológicos tales como las apetencias, el sexo, la edad mental y la fatiga; entendida esta como el decrecimiento del rendimiento laboral por causa del cansancio físico y mental que se presenta por la exposición prolongada al trabajo. Entre más prolongada sea esta exposición, más peligrosa será la condición de trabajo: un conductor que maneja por tiempos muy prolongados, por ejemplo, puede causar y sufrir un accidente grave como producto de su lenta capacidad de reacción y la pobreza en sus reflejos, efectos de su cansancio físico (Cruz \& Garnica, 2010).

En este orden de ideas, podría entenderse que la fatiga es una respuesta generalizada al estrés sostenido en el tiempo y que se compone de tres aspectos: i.) la fatiga fisiológica que redunda en una disminución de la capacidad física del individuo, ii.) la fatiga objetiva que deriva en un detrimento de la productividad, y iii.) la fatiga subjetiva que hace alusión a la aparición de una percepción o sentimiento de cansancio. Adicionalmente, la fatiga laboral depende de las características del entorno en el que se desempeña el individuo y de las demandas que le impongan por las tareas a su cargo. De ahí que existan factores del trabajo que se pueden asociar a una situación de fatiga, entre los cuales se destacan la carga física y la carga mental (BoadaGrau \& Ficapal-Cusí, 2012).

A fin de afianzar estos conceptos es pertinente señalar que, según Santibáñez (2007), la carga de trabajo es el conjunto de requerimientos que debe satisfacer un trabajador para desempeñar adecuadamente su tarea, cuyas características dependen de la exigencia horaria de trabajo, el ambiente psicológico y/o social de la empresa, el puesto de trabajo y la tarea en sí. Ahora bien, esta carga laboral se puede estudiar desde el componente muscular, denominado carga muscular de trabajo, o desde las exigencias psíquicas y sensoriales a las que se les atribuye el término carga mental de trabajo. El primer componente aborda los esfuerzos físicos en que los músculos realizan contracciones isotónicas que se caracterizan por los cambios en la longitud de cada músculo y que requieren un mayor consumo de energía, y contracciones isométricas en las que el trabajo es estático y se evidencia un aumento de la fuerza muscular sin variaciones en la longitud de los músculos. Igualmente, la carga mental de trabajo se compone de elementos visuales, auditivos, cognitivos, reflexivos y de toma de decisiones. 
Con respecto a la aparición de fatiga en las diferentes ocupaciones, la labor del conductor se destaca por la fuerte relación que existe entre la carga de trabajo y el riesgo de accidente en circulación, pues la fatiga conlleva a incrementar el tiempo de reacción y a disminuir la capacidad visual. Factores como la monotonía en la carretera, el pavimento defectuoso, la lluvia o niebla, las condiciones del vehículo, los largos periodos de conducción, la mala colocación del asiento del vehículo y las velocidades elevadas, influyen en la aparición de la fatiga laboral en los conductores. Como consecuencia, su comportamiento cambia por signos o síntomas tales como el dolor de nuca, adormecimiento en los brazos, cansancio en la espalda, sensación de pesadez en la cabeza, continuos movimientos y cambios de posturas. Esto deriva en situaciones de riesgo por una percepción equívoca de las distancias y formas, dificultad para mantener la atención y el sueño al volante (Navas, 2018).

\section{Materiales y Métodos}

En el sur del Tolima opera la cooperativa de transportadores Cootransplanadas, que cuenta con 144 conductores quienes se dedican al transporte especial colectivo de pasajeros, urbano e intermunicipal. La ejecución del presente proyecto considera el 100\% de dicha población, a fin de realizar una selección y caracterización de cada individuo para identificar el tipo de fatiga laboral que le afecta. Habida cuenta de que la mayoría de los instrumentos utilizados en el proyecto miden cuantitativamente el nivel de fatiga laboral mediante la identificación de síntomas en la población objetivo; se realizó un muestreo probabilístico aleatorio simple y se seleccionaron las siguientes herramientas para la recolección de la información:

1) Prueba de patrones subjetivos de Yoshitake, desarrollada para medir la fatiga asociada al trabajo y que considera tres factores para la valoración (Encarnación, 2016, p.27):

a) Factor tipo 1: trabajos con exigencias mixtas (físicas y mentales).

b) Factor tipo 2: trabajos con exigencias mentales

c) Factor tipo 3: trabajos con exigencias físicas.

2) Cuestionario de hábitos, antecedentes personales y condiciones de trabajo, para ser diligenciado de forma individual y que utiliza para conocer los determinantes individuales de cada trabajador. Para valorar los parámetros cuantitativos, se tienen en cuenta recomendaciones internacionales para evaluación de la Carga física.

3) Evaluación de la carga física: Nota Técnica de Prevención NTP 177 del Instituto Nacional de Seguridad e Higiene en el Trabajo del Ministerio de Trabajo y Asuntos Sociales de España (INSHT, 1987), que sugiere tres criterios para la valoración de carga física:

a) Consumo de energía por medio de la observación de la actividad a desarrollar por el operario, la estimación de todas las operaciones y movimientos elementales y el cálculo consumo energético total.

b) Medida del consumo de oxígeno del operario durante el trabajo, ya que existe una relación lineal entre el volumen de aire respirado y el consumo energético.

c) Análisis de la frecuencia cardiaca para calcular el consumo energético. 
Además, el estudio considera parámetros biomédicos a fin de facilitar la priorización de los conductores que hacen parte de la muestra, según la caracterización del riesgo al que se encuentran expuestos y de acuerdo con sus condiciones de tensión arterial, frecuencia cardiaca, índice de masa corporal e índice cadera-cintura.

\section{Resultados preliminares}

Dado que la fatiga es un fenómeno que puede manifestarse de manera subjetiva u objetiva, para efectos de la investigación es importante recolectar la mayor cantidad de información sobre las manifestaciones de fatiga que se presentan en el grupo de trabajadores participantes. Actualmente el proyecto se encuentra en la fase de ejecución, específicamente en la aplicación de un cuestionario de antecedentes socio-demográficos, a fin de cumplir con los objetivos propuestos en la investigación.

Los instrumentos seleccionados permiten valorar la fatiga desde los diferentes puntos de vista y generar datos para identificar el grado de fatiga que se presenta en el trabajador, los factores de riesgo asociados a sus hábitos de vida y enfermedades comunes. El cuestionario de antecedentes socio-demográficos busca consolidar información relevante sobre el perfil socio-demográfico de la población objeto de estudio, así como identificar los antecedentes de enfermedad de origen común o de origen laboral, estilo de vida y lo más importante, las condiciones en que se desarrolla el trabajo.

En 1987 el Instituto Nacional de salud de los trabajadores INSAT, adaptó y modificó el cuestionario de Fatiga de Yoshitake, diseñado en japón en 1978 (Encarnación, 2016). Este cuestionario, en su versión adaptada para Latinoamérica, consta de 30 preguntas que identifican manifestaciones cognitivas y físicas en el trabajador. Adicionalmente, los interrogantes se agrupan, para clasificar las exigencias del trabajo según sean mentales, físicas y mixtas. Los resultados preliminares de la aplicación de este cuestionario a la muestra de conductores de Cootransplanadas, permiten identificar la fatiga física como un riesgo laboral presente en un alto porcentaje de la población, lo que pude repercutir en su estado de salud a corto, mediano y largo plazo.

Así mismo, la fatiga mixta se evidencia también en una proporción importante de los individuos de la muestra que presentan manifestaciones de agotamiento físico y mental, por lo que se deben considerar las condiciones de trabajo de los conductores y la posibilidad de que estas deriven en incidentes o accidentes laborales. A continuación, se ilustran los porcentajes de fatiga laboral obtenidos de la aplicación del test de Yoshitake a los sujetos de estudio. En la figura 1 se puede apreciar que 62 conductores, esto es el $61,4 \%$ de la muestra estudiada, presentan fatiga física. Sumado a esto, 33 conductores (32,7\%) manifiestan fatiga mixta y 6 individuos (5,9\%) experimentan fatiga mental; de acuerdo con los criterios de Yoshitake. 
Figura 1. Resultados cuestionario de síntomas subjetivos de fatiga de Yoshitake.

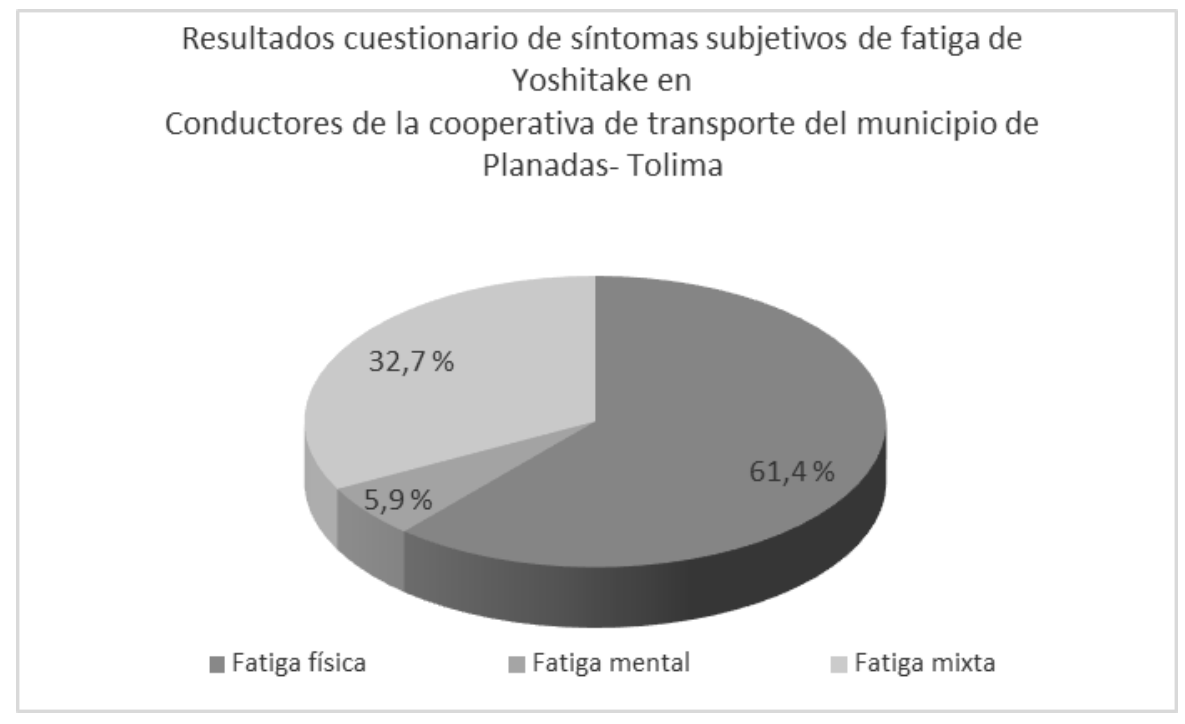

Fuente: Propia (2020)

Por otra parte, el método RULA (Rapid Upper Limb Assessment), validado por la Universidad de Nottingham y publicado en 1993, fue diseñado para medir la exposición a factores de riesgo que puedan afectar tronco, cuello y miembros superiores (Reyes et al., 2013). Mediante su aplicación se pretende establecer la relación entre las posturas prolongadas derivadas de las actividades de conducción en este grupo de trabajadores.

En cuanto a los signos vitales, se consideran parámetros fisiológicos que suelen afectarse por exigencias del entorno tales como el estrés, o por condiciones a las que se somete el cuerpo tales como el esfuerzo, la carga física o la carga mental. Para la recolección de los datos relacionados con estos parámetros se diseñó una matriz que permitió consolidar los datos que, de acuerdo con la Nota Técnica Preventiva 177 del INSHT, pueden ser utilizados para calcular la carga física. Igualmente, se tuvieron en cuenta las recomendaciones de la estrategia Conoce tu riesgo peso saludable del Ministerio de Salud de Colombia (2015), de tal forma que se utilizaron criterios para la identificación de riesgo cardiovascular y diabetes en la muestra seleccionada; a fin de garantizar que las recomendaciones que se dirijan a la población objeto de estudio, al finalizar la presente investigación, estén basadas en el diagnóstico de las condiciones de salud y se orienten a la promoción de estilos de vida saludable y prevención de los factores de riesgo.

A este respecto, la Organización Mundial de la Salud (2008) en la Guía de bolsillo para la estimación y el manejo del riesgo cardiovascular, establece que las enfermedades cardiovasculares corresponden a una de las causas más significativas de enfermedad y muerte en el mundo y que, por tal razón, se deben considerar en la definición, propuesta y programación de medidas y acciones preventivas en las organizaciones. Precisamente, algunos factores de riesgo identificados en los conductores de la Cooperativa de Transportadores de Planadas, tales como el consumo de tabaco o los elevados niveles en la presión arterial y en los índices cadera-cintura y de masa corporal; pueden ser intervenidos para su reducción y control, de modo que se eviten enfermedades cardiacas o patologías crónicas como la diabetes o la hipertensión. 
Evaluación de la fatiga laboral en conductores de la cooperativa de transporte del municipio de Planadas

Tabla 1

Factores de riesgo cardiovascular identificados por grupos de edad, en conductores de la cooperativa de trasportadores de Cootransplanadas

\begin{tabular}{ccccc}
\hline Edad & Población & $\begin{array}{c}\text { Presión arterial sistólica } \\
\text { por encima de } 140\end{array}$ & $\begin{array}{c}\text { Índice cadera cintura por } \\
\text { encima de 0,95 }\end{array}$ & $\begin{array}{c}\text { Índice de masa corporal } \\
\text { por encima de 25 }\end{array}$ \\
\hline 20 a 30 años & 20 & 1 & 1 & 16 \\
31 a 40 años & 29 & 6 & 15 & 26 \\
41 a 50 años & 24 & 4 & 19 & 22 \\
51 a 60 años & 22 & 4 & 16 & 3 \\
61 a 70 años & 6 & 0 & 6 & 85 \\
\hline Total, muestra & 101 & 15 & 57 & 3 \\
\hline
\end{tabular}

Fuente: Propia (2020)

La tabla 1, muestra los resultados iníciales de la captura de datos relativos a los parámetros fisiológicos del grupo de trabajadores de Cootransplanadas. Se valoraron 101 conductores mediante la toma de presión arterial, frecuencia cardiaca, saturación de oxígeno, índice de masa corporal e índice cadera cintura. De igual manera, se agruparon los conductores según su edad, entre los 20 y los 70 años, y se encontró que:

a) El mayor grupo de población se encuentra en el rango de edad de 31 a 40 años.

b) Todos los grupos analizados tienen riesgo por índice cadera cintura por encima de 0,95 (56\% de la muestra) e índice de masa corporal por encima de 25 (84\% de la muestra), lo que evidencia un riesgo de obesidad en todos los grupos de edad.

c) El riesgo con menor presencia en el grupo de conductores es el de presión arterial sistólica por encima de 140 (14,8\% de la muestra).

Para finalizar, es de resaltar que todos aquellos trabajadores que registren marcadores de fatiga física se someterán al Test de Harvard, que consiste en una prueba de tolerancia al esfuerzo físico y la capacidad aérobica (Niño, 2010), llamada también prueba escalón, y que puede aportar importantes datos sobre la condición general de los conductores y orientar las medidas de promoción de la salud y prevención de la enfermedad.

\section{Conclusiones}

La fatiga física en conductores es un resultado multifactorial que se genera a partir del desarrollo de las tareas de conducción y de las exigencias que impone el sistema de trabajo, que deriva en un conjunto de trastornos en tejidos blandos tales como tendones, cápsula tendinosa, nervios, y músculos. Asimismo, los horarios extendidos de trabajo, la sobrecarga postural, los hábitos y el sedentarismo del individuo son factores que se encuentran relacionados a la carga física.

Cuando se presenta trabajo estático es necesario evaluar la ubicación espacial de los segmentos corporales y recopilar información a través de observación directa, registro fotográfico o audiovisual y técnicas goniométricas que permiten hallar los grados de desplazamiento de los IPSA SCIENTIA - Vol. 5 No $1-2020$ 
segmentos evaluados. Por esta razón el método Rapid Upeper Limb Asseessment (RULA) es idóneo para valorar la carga postural en el presente estudio, una vez se establezcan las posturas de trabajo y se comparen con valores de referencia para determinar el nivel de riesgo.

De otro lado, la labor de conducción que desarrollan los colaboradores de Cootransplanadas generalmente implica posiciones fijas o restringidas en el cuerpo, que se sostienen en tiempos que oscilan entre 6 y 8 horas en promedio; con trayectos inestables, presencia de vibraciones de cuerpo entero y exposición a condiciones de discomfort térmico. De esta manera, el instrumento de Yoshitake cumple con criterios de idoneidad para la identificación de síntomas de fatiga asociados a la tarea de conducción y permite una eventual correlación de resultados con los datos que se obtengan a partir de la aplicación de otros instrumentos tales como el test de Harvard o el método RULA.

En cualquier caso, la conducción es un trabajo que demanda un porcentaje de carga física importante, originado por movimientos repetitivos de miembros inferiores y superiores, así como la postura prolongada en el vehículo, con sobre esfuerzo ocasional en rodillas, hombros y codos. El sobre esfuerzo puede estar determinado por las características del vehículo tales como el modelo, la capacidad de pasajeros y el estado de mantenimiento de la dirección, la caja de cambios y los pedales, entre otros. Adicionalmente, en las áreas rurales de Colombia los conductores de transporte público deben manipular manualmente objetos pesados en los vehículos durante varios momentos en la ruta, a solicitud de los pasajeros, lo que deriva en aumento del esfuerzo físico y en la posibilidad de sufrir fatiga laboral.

En relación con los parámetros fisiológicos evaluados en los conductores, la posible presencia de enfermedades cardiovasculares o crónicas en la muestra seleccionada, y los factores de riesgo que se detectaron al capturar los datos relacionados con tendencias y hábitos de vida, se espera encontrar una correlación entre la fatiga y el estado de salud del trabajador. En otras palabras, es posible que incremente el riesgo de fatiga laboral, a medida que se presente mayor carga de enfermedad crónica.

Para finalizar, impera destacar que los resultados que se obtienen a partir de la aplicación de los instrumentos descritos y de la información que aporta cada uno de los individuos seleccionados en el objeto práctico de estudio; facilitan el desarrollo de estrategias para aumentar el nivel de desempeño en seguridad y salud en el trabajo y, en consecuencia, contribuyen a la mejora de las condiciones de trabajo de los conductores y seguridad, salud, calidad de vida y trabajo de los conductores.

\section{Referencias}

Boada-Grau, J. \& Ficapal-Cusí, P. (2012). Salud y trabajo: los nuevos y emergentes riesgos psicosociales. Barcelona: Editorial UOC.

Cámara de Comercio de Bogotá. (8 de diciembre de 2020). Región ordenada, conectada y sostenible. https://www.ccb.org.co/observatorio/Region/Region-ordenada-conectada-y-sostenible/Movilidad

Cañas, J. \& Madrid, I. (2015). Ergonomía. En J. L. Moreneo, C. Molina y S. Olarte (Eds.), Tratado de prevención de riesgos laborales. Teoría y práctica (pp. 1 - 74). Tecnos.

IPSA SCIENTIA - Vol. 5 No $1-2020$ 


\section{Evaluación de la fatiga laboral en conductores de la cooperativa de transporte del municipio de Planadas}

Colombia, Ministerio de Salud (2018). Estrategia "Conoce tu Riesgo Peso Saludable”. Instructivo para la utilización de la calculadora de riesgo. Bogotá. Recuperado de https://www.minsalud.gov.co/sites/rid/List s/BibliotecaDigital/RIDE/VS/PP/instructivo-aplicativo-conoce-riesgo.pdf.

Cruz Gómez, A. \& Garnica Gaitán, A. (2010). Ergonomía Aplicada. Bogotá: Eco Ediciones.

Encarnación Villalta, K. (2016). Influencia de posturas inadecuadas en la fatiga laboral de los trabajadores de una Empresa Manufacturera de Quito-Ecuador 2015 (Tesis de maestría). Universidad Central del Ecuador. http://www.dspace.uce.edu.ec/handle/25000/7182

Instituto Nacional de Seguridad e Higiene en el Trabajo. (1987). NTP 177 La carga física de trabajo: definición y evaluación [Archivo PDF]. https://www.insst.es/documents/94886/326801/ntp_177.pdf/83584437-a435-4f77b708-b63aa80931d2

Lerman, S. E., Eskin, E., Flower, D. J., George, E. C., Gerson, B., Hartenbaum, N., Hursh, S. R., \& Moore-Ede, M. (2012). Fatigue Risk Management in the Workplace. Journal of Occupational and Environmental Medicine, 54(2), 231-258. doi: 10.1097/JOM.0b013e318247a3b0

National Highway Traffic Safety Administration. (2016). NHTSA Drowsy Driving Research and Program Plan. https://www.nhtsa.gov/sites/nhtsa.dot.gov/files/drowsydriving_strategicplan_030316.pdf

Navas Cuenca, E. (2018). Prevención de riesgos laborales: sector Servicios: riesgos específicos del trabajo de conductor (2a. ed.). Málaga: Editorial ICB.

Niño Hernández, C. (2010). Evaluación de la aptitud cardio respiratoria. Movimiento Científico, 4(1), 68-72. https://revmovimientocientifico.ibero.edu.co/article/download/286/254/

Organización Mundial de la Salud, (2008). Guía de Bolsillo para la Estimación y el Manejo del Riesgo Cardiovascular. Recuperado de: https://www.who.int/publications/list/cadio_pocket_guidelines/es/

Pachón Florez, C., Barrios Barreto, M. \& Pérez Jiménez, J. (2017). Riesgo ergonómico en los conductores de transporte intermunicipal de Sucre. En Corporación Universitaria Antonio José de Sucre CORPOSUCRE (Ed.), Universidad - Ciencia - Innovación y Sociedad: desde la perspectiva laboral (pp. 49 - 76). Ediciones Universidad Simón Bolivar.

Reyes García, E., Salgado Guadarrama, J., Quintana Vilchis, B. y Pérez Ilagor, V. Aplicación del método RULA (Rapid Upper Limb Assessment) para determinar riesgo ergonómico en enfermeras instrumentistas de un hospital de tercer nivel. Horizontes en Salud: expresión de enfermería, 5(1), 29-38. DOI: 10.13140/RG.2.1.4617.7444

Santibáñez Lara, I. \& Sánchez Vega, J. (2007). Jornada laboral, flexibilidad humana en el trabajo y análisis del trabajo pesado. Buenos Aires: Ediciones Díaz de Santos.

The National Institute for Occupational Safety and Health (NIOSH) Science Blog. (8 de diciembre de 2020). La fatiga relacionada con el trabajo va más allá del ámbito laboral. Atlanta: Newstex. Recuperado de https://search-proquest-com.ezproxy.uniminuto.edu/docview/2404414618?accountid=48797

Vega-Gómez, N., Ceballos-Burgos, H., \& Pardo-Carmona, J. (2020). Riesgos psicosociales en trabajadores calificados por enfermedad laboral frente a trabajadores que esperan por calificación. IPSA Scientia, Revista científica Multidisciplinaria, 5(1), 112-126. https://doi.org/10.25214/27114406.995

IPSA SCIENTIA - Vol. 5 No $1-2020$ 